IMA Journal of Applied Mathematics (2005) 1-17

doi: 10.1093/imamat/dri017

\title{
A Comparative Study of Two Macro-Scale Models of Condensed-Phase Explosives
}

\author{
DONALD W. SCHWENDEMAN AND ASHWANi K. KAPILA \\ Department of Mathematical Sciences, Rensselaer Polytechnic Institute, \\ Troy, New York, 12180, USA. \\ WiLliam D. HENSHAW \\ Center for Applied Scientic Computing, Lawrence Livermore National Laboratory, \\ Livermore, California, 94550, USA.
}

\begin{abstract}
Two macro-scale models of high-energy, condensed-phase explosives are considered. The first, called ignition and growth, treats the heterogeneous explosive as a homogeneous mixture of reacting and product species. The second model treats the explosive as two distinct, but interacting, phases. Both models are hyperbolic systems of nonlinear partial differential equations involving balance laws of mass, momentum and energy, and involving assumed constitutive input and reaction kinetics. The two-phase model also involves stiff relaxation terms and nozzling terms that prevent the equations from being cast in conservation form. A high-resolution shock-capturing scheme on overlapping grids, with parallel adaptive mesh refinement, is used to solve the governing equations accurately. The purpose of the paper is to compare solutions of the two models for macro-scale problems involving detonation initiation and diffraction. This is done to illustrate strengths and weaknesses of the models, and to suggest modifications and extensions of the models for further study.
\end{abstract}

Keywords: reactive flow, detonation, Euler equations, Godunov methods, corner turning, diffraction.

\section{Introduction}

High-energy, condensed-phase explosives are heterogeneous materials with a complex microstructure consisting of crystalline grains of the energetic material held together by an inert plastic binder. In addition, the microstructure typically contains voids, gaseous pores, metallic particles and other impurities. When an explosive is subjected to a shock of sufficient strength, a detonation (a shock supported by chemical energy) is initiated. Although the crystalline homogeneous explosive has a high ignition threshold, relatively weaker stimuli are known to be sufficient to initiate the heterogeneous explosive. This propensity is attributed to a nonuniform temperature field that occurs when the granular material is exposed to the initiating shock. Discrete sites, where the local temperature is much higher than the bulk average, are generated as a result of mechanical processes including friction, pore collapse, shear banding and local plastic deformation. These sites, or hot spots, act as preferred locations of ignition, where burning commences and then spreads to consume the entire bulk. When averaged over a sufficiently large scale, this heterogeneous behavior produces an average heat release rate that is a combination of chemical kinetics at the molecular scale and mechanics at the grain scale. A rational description of this multi-scale behavior would require an understanding of the underlying processes at each relevant scale, followed by a procedure of porting this information across the scales to produce a mathematical model appropriate for the scale of observation. Such a multi-scale description is not available at the present time, and instead various phenomenological continuum models have been developed to simulate detonation behavior at the macro (lab) scale. 
Mathematical models of macro-scale behavior of condensed-phase explosives are more or less complex depending on the level of homogenization assumed (either explicitly or implicitly) in their continuum descriptions. One class of models considers the explosive to be a homogeneous mixture of reacting and product species in local mechanical and thermal equilibrium. A single reaction progress variable (the mass fraction of product species) is introduced to measure the composition of the mixture at any point in space and time. The state variables for the mixture are assumed to satisfy balance laws of mass, momentum and energy, together with an assumed rate equation for the reaction progress variable. An example of this type of homogeneous mixture model is the well-studied ignition-and-growth (IG) model, which was proposed originally by Lee \& Tarver (1980) and later refined and utilized by Tarver and colleagues (see Tarver (2005) and the references cited therein). A second class of models assumes that the reacting and product species retain their constitutive identities so that the explosive is treated as a two-phase continuum. Balance laws are assumed for each phase separately, and these equations include source terms representing the exchange of mass, momentum and energy across the interfacial boundaries between phases. Both phases are taken to be compressible due to the very high pressure in the system behind the detonating shock. An assumed compaction law and a saturation constraint complete the set of equations for the model. An example of this latter continuum description is the well-known two-phase model discussed by Baer \& Nunziato (1986).

The equations governing the IG and two-phase models are hyperbolic systems of nonlinear partial differential equations which may or may not possess a conservation form. These equations require numerical methods to obtain solutions for various initial conditions and flow configurations. In addition to the usual difficulties associated with discontinuities in the flow (at shocks and contacts), solutions of interest have thin zones in space and time in which the state variables change rapidly due to chemical reaction and/or interphase relaxation. Experience has shown that these thin zones must be adequately resolved in order to obtain solutions accurately. Adaptive mesh refinement (AMR) is essential to compute well-resolved solutions efficiently, and parallel computing is necessary to obtain accurate fine-grid results for simulations in two and three space dimensions. Various numerical methods have been developed for macro-scale models with these considerations in mind. The approach used here employs a high-resolution Godunov-type shock-capturing method on overlapping grids. The basic method with AMR for the reactive Euler equations is described in Henshaw \& Schwendeman (2003), and an extension for parallel computing is given in Henshaw \& Schwendeman (2008). An application of the basic approach for the IG model is discussed in Kapila et al. (2007) for rigid confinement and in Banks et al. $(2007,2008)$ for compliant confinement. An extension of the approach for the equations governing two-phase flow is described in Schwendeman et al. $(2006,2007)$ for one-dimensional problems and in Schwendeman et al. (2010) for two-dimensional problems. Other numerical methods are available for macro-scale models of the types considered here, and a discussion of these methods may be found in the references provided in the aforementioned papers.

Assuming that a numerical approach is available to compute solutions accurately, a critical assessment of a macro-scale model can be made by exploring solution behaviors for a range of problem configurations and by comparing solutions with available experimental data. Of particular interest is the ability of the model to accurately simulate dynamic phenomenon at the macro scale. Such phenomenon include detonation initiation, detonation propagation and diffraction, and detonation failure. For the case of detonation diffraction at a $90^{\circ}$ corner, for example, it is known that pockets of unburnt explosive can appear behind the leading shock due to a failure of the detonation in the post-diffracted region. This macro-scale behavior, resulting in so-called dead zones, has received attention in the literature (see, for example, the work in Ferm et al. (2001), Souers et al. (2002) and Tarver (2005)), and has been the focus of our recent work in Kapila et al. (2007) and Banks et al. (2008) for the IG model and in Schwende- 
man et al. (2010) for the two-phase model. The purpose of this paper is to briefly describe this recent work and to make a critical comparison of the behavior of solutions given by the two models for onedimensional detonation initiation and two-dimensional detonation diffraction. Such a comparison sheds light on the strengths and weaknesses of the two models, and gives an indication of how the models might be modified or extended to better simulate detonation behavior at the macro scale.

The remaining sections of the paper are organized as follows. In Section 2, we describe the modeling assumptions and governing equations for the IG and two-phase models. This discussion is followed in Section 3 by a brief discussion of the adaptive numerical approach used to compute accurate solutions of the models on overlapping grids. A comparison of numerical simulations of detonation initiation and diffraction is discussed in Section 4, and concluding remarks are made in Section 5.

\section{Mathematical models}

In this section, we provide a brief description of the two macro-scale models starting with the IG model in Section 2.1 followed by the two-phase model in Section 2.2.

\subsection{Ignition and growth}

The IG model, originally derived by Lee \& Tarver (1980) and later refined by Tarver and colleagues, treats the heterogeneous explosive as a homogeneous mixture of two constituents, the unreacted explosive and the reaction products. The consequences of microstructural heterogeneity, not reflected in the thermo-mechanical description, are accounted for in an impressionistic way in the formulation of the reaction rate. Separate Jones-Wilkins-Lee (JWL) equations of state are prescribed for each constituent (see Fickett \& Davis (1979)). These equations have the following mechanical and thermal forms:

$$
e_{i}=e_{i}\left(p_{i}, \rho_{i}\right), \quad p_{i}=p_{i}\left(T_{i}, \rho_{i}\right), \quad i=s \text { or } g
$$

where $e_{i}$ is the specific internal energy, $p_{i}$ is the pressure, $\rho_{i}$ is the density and $T_{i}$ is the temperature of constituent $i$, either the unreacted solid explosive $s$ or the gaseous products of reaction $g$. The following mixture rules define the specific energy $e$ and specific volume $1 / \rho$ of the mixture in terms of the corresponding quantities for the constituents and the reaction progress variable $\lambda$ (the mass fraction of the product):

$$
e=(1-\lambda) e_{s}+\lambda e_{g}, \quad 1 / \rho=(1-\lambda) / \rho_{s}+\lambda / \rho_{g}
$$

It is assumed that both constituents move with the same velocity and that they are in pressure and temperature equilibrium, i.e. $p_{s}=p_{g}=p$ and $T_{s}=T_{g}=T$. An equation of state for the mixture of the form $e=e(\rho, p, \lambda)$ can be constructed using the equations of state in (2.1) and the mixture rules in (2.2) together with the assumption of pressure and temperature equilibrium (see Kapila et al. (2007) for further details).

It is further assumed that the mixture variables satisfy the reactive Euler equations. In two dimensions these equations are

$$
\frac{\partial}{\partial t} \mathbf{u}+\frac{\partial}{\partial x_{1}} \mathbf{f}_{\mathbf{1}}(\mathbf{u})+\frac{\partial}{\partial x_{2}} \mathbf{f}_{\mathbf{2}}(\mathbf{u})=\mathbf{g}(\mathbf{u})
$$


where

$$
\mathbf{u}=\left[\begin{array}{c}
\rho \\
\rho v_{1} \\
\rho v_{2} \\
\rho E \\
\rho \lambda
\end{array}\right], \quad \mathbf{f}_{1}=\left[\begin{array}{c}
\rho v_{1} \\
\rho v_{1}^{2}+p \\
\rho v_{1} v_{2} \\
v_{1}(\rho E+p) \\
\rho v_{1} \lambda
\end{array}\right], \quad \mathbf{f}_{2}=\left[\begin{array}{c}
\rho v_{2} \\
\rho v_{1} v_{2} \\
\rho v_{2}^{2}+p \\
v_{2}(\rho E+p) \\
\rho v_{2} \lambda
\end{array}\right], \quad \mathbf{g}=\left[\begin{array}{c}
0 \\
0 \\
0 \\
0 \\
\rho \mathscr{R}
\end{array}\right] .
$$

Here, $\left(v_{1}, v_{2}\right)$ is the velocity, $\mathscr{R}$ is the reaction rate and $E=e+\frac{1}{2}\left(v_{1}^{2}+v_{2}^{2}\right)$ is the total energy per unit mass.

The chemical reaction rate equation $\mathscr{R}$ in the IG model is a pressure-driven rule of the form

$$
\begin{aligned}
\mathscr{R}=I & (1-\lambda)^{b}\left(\rho / \rho_{0}-1-a\right)^{x} H\left(\lambda_{\mathrm{ig}, \max }-\lambda\right) \\
& +G_{1}(1-\lambda)^{c} \lambda^{d} p^{y} H\left(\lambda_{G_{1}, \max }-\lambda\right)+G_{2}(1-\lambda)^{e} \lambda^{g} p^{z} H\left(\lambda-\lambda_{G_{2}, \min }\right),
\end{aligned}
$$

where $\rho_{0}$ is the ambient density of the solid explosive, $H$ is the Heaviside function, and $I, G_{1}, G_{2}, a, b$, $c, d, e, g, x, y$ and $z$ are constants. Three switching constants, $\lambda_{\mathrm{ig}, \max }, \lambda_{G_{1}, \max }$ and $\lambda_{G_{2}, \min }$ also appear, and limit the contributions of the three terms to, respectively, a maximum reacted fraction $\lambda_{\mathrm{ig}, \mathrm{max}}$ for the ignition term, a maximum reacted fraction $\lambda_{G_{1}, \max }$ for the first growth term, and a minimum reacted fraction $\lambda_{G_{2}, \text { min }}$ for the second growth term. The constant $a$ in the first term defines a compression threshold that must be exceeded before ignition will occur. The exponents $b, c, d, e$ and $g$ are topological constants that mimic the grain-burning configuration. The pressure exponents $y$ and $z$ are measures of the rate sensitivity of the reaction rate. The three terms are designed to model the three stages of reaction that are believed to occur in shock initiation and propagation of detonations in heterogeneous explosives.

The IG model involves a large set of parameters whose values are chosen based on calibrations with experiments. For example, the equation of state for the reactant is fitted to available shock-Hugoniot data and the product equation of state is fitted to data from cylinder tests and other metal acceleration experiments. The parameters for the reaction rate are fitted to experimental measurements involving detonation initiation and propagation (see Tarver (2005) and Kapila et al. (2007)).

\subsection{Two phase}

We now consider a model that treats the solid reactant and the gaseous product as distinct phases. The equations for this model consist of balance laws of mass, momentum and energy for each phase separately, plus an equation that allows compaction of the solid phase driven by the pressure difference between the phases. Terms representing interfacial exchange of mass, momentum and energy appear, corresponding to the nonequilibrium processes of reaction, drag and heat transfer. Several models of this kind have been proposed in the literature. The pioneering work is that due to Baer \& Nunziato (1986), who formulated the first two-phase continuum description of high-energy explosive materials. Also noteworthy are the contemporaneous study by Butler \& Krier (1986), and the later work by Powers et al. (1990a,b). A critical examination of the model and a study of various reduced sub-models is discussed by Bdzil et al. (1999), Kapila et al. (1997) and Kapila et al. (2001). Also, similar models have been proposed by Saurel and collaborators; see Saurel \& Abgrall (1999) and Saurel \& Lemetayer (2001), and the extensive bibliographies therein.

For the present discussion, we consider the two-phase Baer-Nunziato model as modified in Bdzil et al. (1999). In two dimensions the governing equations for this model may be written in the form

$$
\frac{\partial}{\partial t} \mathbf{u}+\frac{\partial}{\partial x_{1}} \mathbf{f}_{1}(\mathbf{u})+\frac{\partial}{\partial x_{2}} \mathbf{f}_{2}(\mathbf{u})=\mathbf{h}_{1}(\mathbf{u}) \frac{\partial \bar{\alpha}}{\partial x_{1}}+\mathbf{h}_{2}(\mathbf{u}) \frac{\partial \bar{\alpha}}{\partial x_{2}}+\mathbf{k}(\mathbf{u}),
$$


where

$$
\begin{gathered}
\mathbf{u}=\left[\begin{array}{c}
\bar{\alpha} \\
\bar{\alpha} \bar{\rho} \\
\bar{\alpha} \bar{\rho} \bar{v}_{1} \\
\bar{\alpha} \bar{\rho} \bar{v}_{2} \\
\bar{\alpha} \bar{\rho} \bar{E} \\
\alpha \rho \\
\alpha \rho v_{1} \\
\alpha \rho v_{2} \\
\alpha \rho E
\end{array}\right], \quad \mathbf{f}_{1}=\left[\begin{array}{c}
0 \\
\bar{\alpha} \bar{\rho} \bar{v}_{1} \\
\bar{\alpha}\left(\bar{\rho} \bar{v}_{1}^{2}+\bar{p}\right) \\
\bar{\alpha} \bar{\rho} \bar{v}_{1} \bar{v}_{2} \\
\bar{\alpha} \bar{v}_{1}(\bar{\rho} \bar{E}+\bar{p}) \\
\alpha \rho v_{1} \\
\alpha\left(\rho v_{1}^{2}+p\right) \\
\alpha \rho v_{1} v_{2} \\
\alpha v_{1}(\rho E+p)
\end{array}\right], \quad \mathbf{f}_{2}=\left[\begin{array}{c}
0 \\
\bar{\alpha} \bar{\rho} \bar{v}_{2} \\
\bar{\alpha} \bar{\rho} \bar{v}_{1} \bar{v}_{2} \\
\bar{\alpha}\left(\bar{\rho} \bar{v}_{2}^{2}+\bar{p}\right) \\
\bar{\alpha} \bar{v}_{2}(\bar{\rho} \bar{E}+\bar{p}) \\
\alpha \rho v_{1} \\
\alpha \rho v_{1} v_{2} \\
\alpha\left(\rho v_{2}^{2}+p\right) \\
-\bar{v}_{1} \\
0 \\
p \\
0 \\
\bar{v}_{1} p \\
0 \\
-p \\
0 \\
-\bar{v}_{1} p
\end{array}\right], \quad\left[\begin{array}{c}
\alpha v_{2}(\rho E+p) \\
0 \\
0 \\
p \\
\bar{v}_{2} \\
0 \\
0 \\
-p \\
-\bar{v}_{2} p
\end{array}\right], \quad \mathbf{k}=\left[\begin{array}{c}
\mathscr{C} / \bar{\rho} \\
\mathscr{C} \\
\mathscr{M}_{1} \\
\mathscr{M}_{2} \\
\mathscr{E}-p \mathscr{F}_{2} \\
-\mathscr{C} \\
-\mathscr{M}_{1} \\
-\mathscr{M}_{2} \\
-\mathscr{E}+p \mathscr{F}
\end{array}\right]
\end{gathered}
$$

Here, $\alpha, \rho,\left(v_{1}, v_{2}\right), p$ and $E$ denote the volume fraction, density, velocity, pressure and the total energy, respectively. Overbars are used to indicate solid phase variables while gas phase variables have no overbars. The total energies are given by

$$
E=e+\frac{1}{2}\left(v_{1}^{2}+v_{2}^{2}\right), \quad \bar{E}=\bar{e}+\frac{1}{2}\left(\bar{v}_{1}^{2}+\bar{v}_{2}^{2}\right)
$$

where $e$ and $\bar{e}$ are specific internal energies. The latter quantities are specified by equations of state for each phase. For example,

$$
e=\frac{p}{(\gamma-1) \rho(1+b \rho)}, \quad \bar{e}=\frac{\bar{p}+\bar{\gamma} \pi}{(\bar{\gamma}-1) \bar{\rho}}+\bar{B}(\bar{\alpha})+\bar{q}
$$

where $\gamma$ and $\bar{\gamma}$ are ratios of specific heats, $b$ is a virial gas coefficient, $\bar{\pi}$ is a constant stiffening pressure for the solid phase, $\bar{q}$ is the heat release associated with the conversion of solid explosive to gaseous product, and $\bar{B}(\bar{\alpha})$ is the compaction potential. The compaction potential accounts for the configurationdependent energy of the solid (see Bdzil et al. (1999)), and the specific choice used here follows that in Schwendeman et al. (2007, 2010). The system is closed by including the saturation constraint $\alpha+\bar{\alpha}=1$.

Source terms representing interactions between the phases appear on the right-hand side of the governing equations. The terms given in $\mathbf{h}_{i}(\mathbf{u}) \bar{\alpha}_{x_{i}}, i=1$ and 2 , are the so-called nozzling terms. They arise naturally in the model and describe momentum and energy exchange between the phases as the result of an effective change in the cross-sectional area of a virtual stream tube in the gas phase. The terms in $\mathbf{k}(\mathbf{u})$ represent compaction, $\mathscr{F}$, compaction work, $p \mathscr{F}$, and an exchange of mass, momentum and energy between phases given by $\mathscr{C},\left(\mathscr{M}_{1}, \mathscr{M}_{2}\right)$ and $\mathscr{E}$, respectively. A number of choices for $\mathscr{C}$ can be made depending on the assumed kinetics of the chemical reactions. Following Baer \& Nunziato (1986), one possible choice is

$$
\mathscr{C}=-\sigma \bar{\alpha} \bar{\rho}\left(p-p_{\text {ign }}\right)^{z}
$$


where $\sigma>0$ is a rate constant, $p_{\text {ign }}$ is an ignition pressure and $z$ is a constant pressure exponent. The other quantities in $\mathbf{k}(\mathbf{u})$ are assumed to have the form

$$
\begin{gathered}
\mathscr{F}=\alpha \bar{\alpha}(\bar{p}-p-\bar{\beta}) / \mu_{c}, \quad \mathscr{M}_{i}=\bar{v}_{i} \mathscr{C}+\left(\delta+\frac{1}{2} \mathscr{C}\right)\left(v_{i}-\bar{v}_{i}\right), \\
\mathscr{E}=(\bar{E}+\beta / \bar{\rho}) \mathscr{C}+\left(\delta+\frac{1}{2} \mathscr{C}\right)\left(\left(v_{1}-\bar{v}_{1}\right) \bar{v}_{1}+\left(v_{2}-\bar{v}_{2}\right) \bar{v}_{2}\right)+\mathscr{H}(T-\bar{T}),
\end{gathered}
$$

where $\bar{\beta}=\bar{\alpha} \bar{\rho} \bar{B}^{\prime}(\bar{\alpha})$ is the configuration pressure, $\mu_{c}$ is the compaction viscosity, $\delta$ is the drag coefficient, $\mathscr{H}$ is the heat-transfer coefficient, and $T$ and $\bar{T}$ are the temperatures of the gas and solid, respectively.

The two-phase model also requires values for several parameters. These include parameters for the equations of state in (2.6), which are taken to be extensions of ideal equations of state unlike the more complicated JWL forms for the IG model. In addition, a choice of parameter values is needed for the pressure-dependent reaction rate in (2.7) and for the exchange source terms in (2.8). The two-phase model has not been subjected to the same level of calibration as compared to the IG model, and so less information is available in the literature regarding choices for the parameters. Following the approach used in Schwendeman et al. (2007), values for the parameters in the equations of state are chosen to match sound speeds and steady detonation velocities for a typical PBX-type explosive. Values for the parameters in the exchange source terms are chosen based on the available estimates found in the literature (see Schwendeman et al. (2007) and the references cited therein).

\section{Adaptive numerical approach}

The equations governing the IG and two-phase models are hyperbolic systems of nonlinear partial differential equations. These two systems can be written in the general form

$$
\frac{\partial}{\partial t} \mathbf{u}+\frac{\partial}{\partial x_{i}} \mathbf{f}_{i}(\mathbf{u})=A_{i}(\mathbf{u}) \frac{\partial}{\partial x_{i}} \mathbf{u}+\mathbf{b}(\mathbf{u})
$$

with Einstein summation convention used. Here, $\mathbf{u}$ denotes the state variables, $\mathbf{f}_{i}$ the conserved fluxes, $A_{i} \mathbf{u}_{x_{i}}$ the non-conservative nozzling terms (for the two-phase model), and $\mathbf{b}$ the non-differentiated source terms. Our numerical approach for (3.1) is a high-resolution shock-capturing method on overlapping grids. The basic approach with AMR is described in Henshaw \& Schwendeman (2003) for the reactive Euler equations, and its extension to parallel computing is discussed in Henshaw \& Schwendeman (2008). Application of the numerical approach is described in Kapila et al. (2007) and Banks et al. $(2007,2008)$ for the IG model and in Schwendeman et al. $(2006,2007,2010)$ for the two-phase model. Since the numerical method is discussed in detail elsewhere, we provide only a brief summary of the approach here.

An overlapping grid consists of a set of structured curvilinear component grids that together cover the domain of interest and overlap where they meet. Each component grid is defined by a mapping from physical space to computational space, the unit square for two-dimensional problems, and an exact transformation of (3.1) is made to this unit computation domain. The numerical solution of the mapped equations is advanced on each component grid using a second-order slope-limited extension of Godunov's method. For the two-phase model, solutions of the associated Riemann problems for Godunov's method are used to evaluate the non-conservative nozzling terms. The non-differentiated source terms are integrated numerically using a second-order Runge-Kutta error-control scheme. The numerical solution on the boundaries of each component grid is obtained either by interpolation at grid overlaps or by boundary conditions at physical boundaries. 


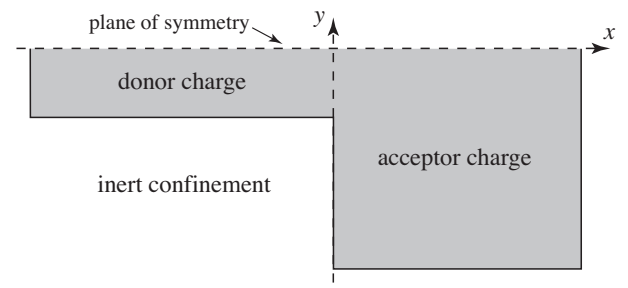

FIG. 1. Geometric configuration of the corner-turning problem.

The overlapping grid covering the domain of interest (such as that shown in Figure 1) forms the base grid for AMR. Refinement grids are built on top of the base grid in a hierarchical fashion according to an estimate of the error. The solution in the vicinity of shocks and contact discontinuities is represented on the finest grid level, and refinement grids are also generated near sharp layers of reaction and/or interphase relaxation, so that a well-resolved numerical solution on the grid is obtained economically. In addition, each grid in the AMR system may be partitioned and computed in parallel. The partitioning of the grids and their distribution amongst a set of processors is done using a load-balancing algorithm. The parallel, AMR approach provides good speed up of the calculations, so that well-resolved solutions are obtained for the results discussed below.

\section{Corner-turning simulations}

In this section we describe numerical simulations of detonation initiation and diffraction. The geometry of the problem is shown in Figure 1. The explosive consists of two sections, a donor charge to the left and an acceptor charge to the right, which are connected at $x=0$. A plane of symmetry for this two-dimensional configuration exists at $y=0$. The explosive is bounded by an inert confining material, which for the present discussion is taken to be rigid. (The case of compliant confinement is discussed in Section 5.) A detonation is initiated in the donor charge at the far left and it propagates to the right towards the acceptor charge. Upon entering the acceptor charge, the detonation wave diffracts as it turns the $90^{\circ}$ corner. The leading shock of the diffracted wave weakens in the acceptor charge due to geometric expansion. The rate of reaction behind the weakened shock slows, and the detonation may fail if the rate of reaction becomes too slow so that the reaction zone separates from the leading shock. Our primary focus here is on the evolution to detonation and on the behavior of the diffracted detonation as determined by the two models.

Experimental and numerical studies of detonation diffraction and corner turning exist already in the literature. Experimental investigations of corner turning have been carried out by Ferm et al. (2001), Souers et al. (2002) and Tarver (2005), among others, for various explosives and geometric configurations. (The configuration shown in Figure 1 follows the work in Ferm et al. (2001).) These studies indicate that detonation failure and dead zones can occur in the post-diffracted region for PBX-type explosives. Numerical studies of detonation diffraction for rigidly confined corner-turning configurations appear in Kapila et al. (2007) for the IG model and in Schwendeman et al. (2010) for the two-phase model. For the IG model, it was found that while the detonation may fail temporarily in the postdiffracted region, the unreacted material reignites either by a process of self-ignition or by the passage of a laterally propagating detonation. In contrast to this behavior, it was found that a sustained dead zone can form for certain choices of the rate parameters of the two-phase model. While detailed discussions 
of the behavior of the detonation for various corner-turning configurations may be found in Kapila et al. (2007) and Schwendeman et al. (2010), a head-to-head comparison of the behaviors determined by the IG and two-phase models has not been made, and this is the main objective of the discussion in this section. Before embarking on such a comparison, it should be noted that the aim here is not to attempt to match parameters of the models so that the solution behaviors are as close as possible, but rather to compare the behaviors for representative parameter sets used in the literature. The idea is to highlight and compare various basic features of the solutions so that strengths and weaknesses of the models can be identified. This exercise may then lead to suggestions for modifications or extensions of the models, which in turn may prompt further investigations.

\subsection{Evolution to steady planar detonation}

The corner-turning problem described above can be considered in two stages. The first stage involves the formation of a steady Chapman-Jouguet detonation wave in the donor charge, while the second stage involves the diffraction of the detonation wave in the acceptor charge. We begin here with a comparison of numerical simulations for the first stage; a discussion of the second stage is given in Section 4.2 below.

Following our previous work, we consider the evolution to detonation due to the impact of a piston with a given velocity $V_{p}$ at the left end of the donor charge. For the IG case, we choose $V_{p}=1075.2 \mathrm{~m} / \mathrm{s}$ which corresponds to the low-speed case discussed in Section 7.1 of Kapila et al. (2007). The parameter set used for the IG model is the "detonation propagation" set given in Tarver (2005) for the explosive LX-17. The plots on the left of Figure 2 show the behavior of the pressure $p$ for the homogeneous mixture and the reaction progress variable $\lambda$. The latter quantity is zero for the unreacted solid and one for the gaseous product of combustion. In these plots, $x$ measures distance from the piston face in the reference frame of the piston. The sudden impact of the piston at $t=0$ generates a shock that propagates to the right away from the piston face. The strength of the shock is sufficient to raise the density $\rho$ behind it so that the fractional change $\rho / \rho_{0}-1$ is greater than the compression threshold $a=0.22$ of the ignition reaction in (2.4). As a result, the reaction turns on behind the shock. The rate of reaction is slow at first as seen by the low values of $\lambda$ at early times. As time increases, the rate of reaction also increases to consume more of the explosive, especially near the piston face. The pressure increases near the piston face as a result of the growing reaction there, and a pressure pulse is generated which moves away from the piston face. The pressure pulse strengthens to form a detonation behind the leading shock, which is well established by the final time $t=1.7 \mu \mathrm{s}$ shown in the plots on the left. Eventually, the detonation overtakes the leading shock, and a steady detonation is established. Further details of this evolution and a discussion of a second case for a larger value for $V_{p}$ may be found in Kapila et al. (2007).

The plots on the right of Figure 2 show a representative simulation of the evolution to detonation for the two-phase model. This calculation uses $V_{p}=1600 \mathrm{~m} / \mathrm{s}$ and the parameter set given by Case II in Schwendeman et al. (2010). For the two-phase model, the pressure $\bar{p}$ of the solid phase and pressure $p$ of the gas phase are both computed as part of the solution. For purposes of comparison, these may be combined to define a mixture pressure $p_{m}$ given by

$$
p_{m}=\bar{\alpha} \bar{p}+(1-\bar{\alpha}) p
$$

where $\bar{\alpha}$ is the volume fraction of the solid phase. This mixture pressure is shown in the upper-right plot of Figure 2, and its behavior may be compared with that given by the single (mixture) pressure $p$ obtained from the solution of the IG model and shown in the plot next to it. For both calculations, 

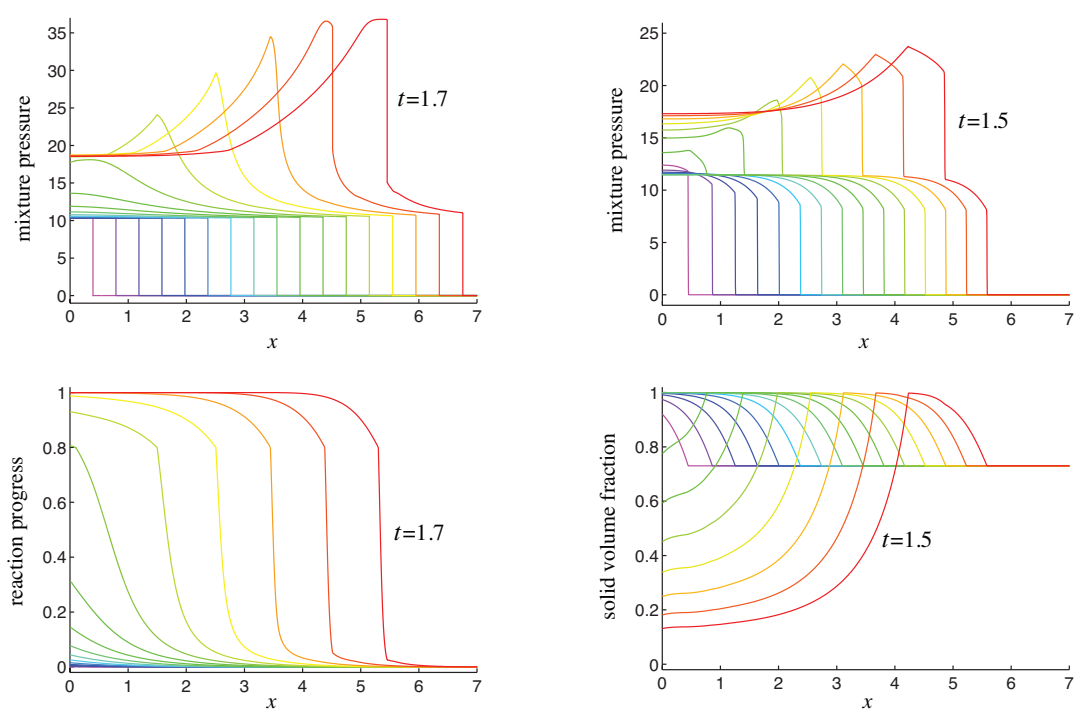

FIG. 2. Evolution to detonation for the IG model (left) and the two-phase model (right). Pressures are given in GPa and distance is given in $\mathrm{mm}$. The time interval between solution curves is $0.1 \mu \mathrm{s}$.

the impact generates a shock which travels to the right. For the two-phase case, a compaction layer described by an increase in $\bar{\alpha}$ can be identified behind the shock. This compaction of the solid grains, whose leading edge moves with the shock, squeezes the gas and increases its pressure to a value above the ignition-pressure $p_{\text {ign }}$ of the two-phase reaction rate in (2.7). The reaction first turns on at the piston face which causes $\bar{\alpha}$ to decrease and $p_{m}$ to increase there. This increase in pressure is similar to that observed for the IG case. The increasing rate of reaction leads to the formation of a pressure pulse and the generation of a secondary shock in the compaction layer behind the leading shock. Although it is not clear from the plot of $p_{m}$, a closer inspection of $p$ and $\bar{p}$ individually (not shown) reveals that the leading shock is a shock in the solid phase while the secondary shock behind it is a shock in the gas phase. This is an example of the level of detail available in the solution of the two-phase model, which provides a richer description of the evolution beyond that given by the simpler IG model. By the final time $t=1.5 \mu$ s shown in the figure, the secondary shock is well established and is moving with a velocity sufficient to overtake the leading shock. A steady detonation wave (shown below) is established at a later time after the secondary shock with the reaction zone behind it has overtaken the leading shock created by the piston impact.

Figure 3 shows the steady detonation profiles for the two models with their chosen parameter sets. The plot in the upper left shows the pressure $p$ and reaction progress $\lambda$ for the detonation profile given by the IG model, while the plot in the lower left shows the corresponding behavior of the three terms in the IG reaction rate in (2.4). For these plots, distance is measured from the leading shock of the detonation and pressure is scaled by $p_{\max }$ (equal to $35.0 \mathrm{GPa}$ for the IG case). The left edge of the steady profile is taken to be the position where $\lambda=0.99$. The profiles for $p$ and $\lambda$ in the reaction zone behind the leading shock of the steady detonation consist of three parts corresponding to the three stages of the IG reaction rate. The first part occurs immediately behind the leading shock where there is a very 

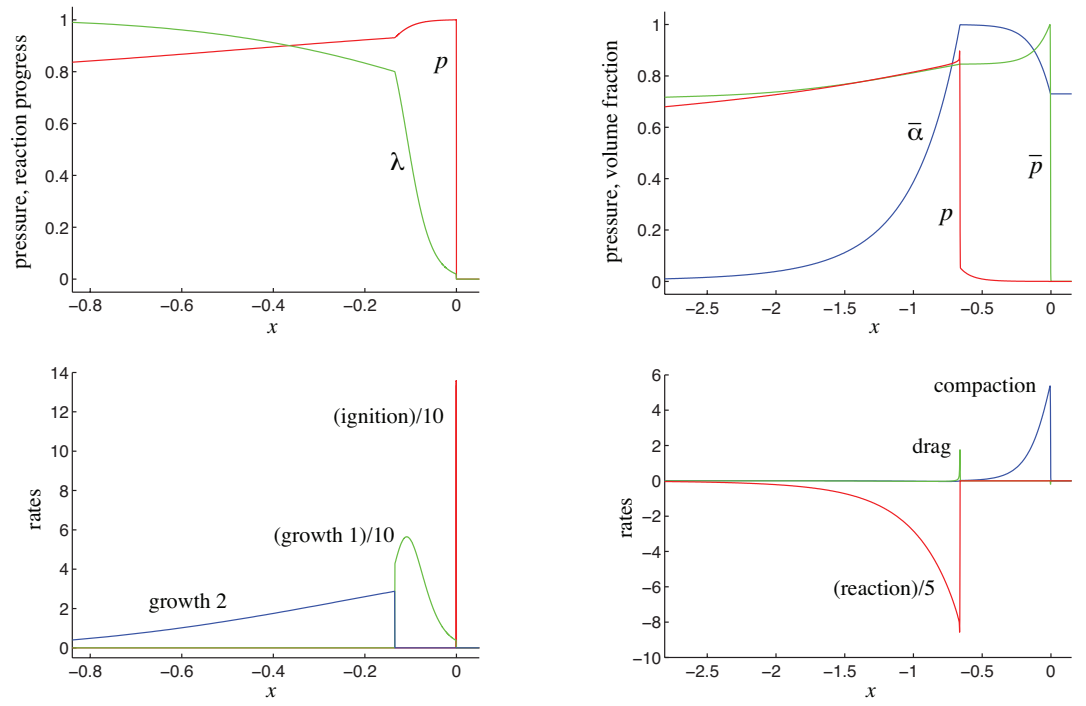

FIG. 3. Steady detonation profiles for the IG model (left) and the two-phase model (right). Pressures are scaled by $p_{\max }$ (equal to $35.0 \mathrm{GPa}$ for the IG case and $29.6 \mathrm{GPa}$ for the two-phase case). Distance is measured in $\mathrm{mm}$ from the leading edge of the detonation. Rates are given in $(\mu \mathrm{s})^{-1}$. (The IG ignition and first growth rates are scaled by 10 and the two-phase reaction rate is scaled by 5 for illustrative purposes.)

narrow interval of $x$ over which the ignition reaction is turned on. The rate of this reaction of very high due to the very large value for the rate constant $I$, but it turns off quickly when $\lambda=\lambda_{\mathrm{ig} \text {,max }}=0.02$ (for the LX-17 parameter set). The majority of the solid explosive is consumed in the second part of the profile where the first growth term is active for $\lambda \leqslant \lambda_{G_{1}, \max }=0.8$. The consumption of explosive occurs slowly over the third part of the profile for $\lambda \geqslant \lambda_{G_{2}, \min }=0.8$ due to the relatively small value for $G_{2}$ for the second growth term. (See Tarver (2005) and Kapila et al. (2007) for a further interpretation of these three terms.) Throughout the steady profile, the pressure decreases monotonically from its peak value at the von Neumann state just behind the leading shock. The kinks in the profile of pressure occur at the switches between terms in the IG reaction rate (most noticeable at the switch between the first and second growth terms).

The behavior of the solid-phase pressure $\bar{p}$, the gas-phase pressure $p$ and the volume fraction of the solid phase $\bar{\alpha}$ for the steady detonation profile of the two-phase model is shown in the upper-right plot of Figure 3. The leading shock of the steady profile is marked by a jump in the pressure of the solid phase. This increase in pressure creates a layer of compaction behind the leading shock which serves to increase the gas-phase pressure. The leading edge of the reaction zone occurs at the end of the compaction layer and is marked by a shock in the gas phase as seen by the jump in the profile for $p$. Behind the shock in the gas phase, the profile of $\bar{\alpha}$ decreases due to the consumption of the solid explosive by the reaction. The left edge of the steady structure shown in the plot is given by the position were $\bar{\alpha}=0.01$. The plot in the lower right of Figure 3 shows the behavior of the reaction rate $\mathscr{C}$ defined in (2.7), the compaction rate $\mathscr{F}$ defined in (2.8), and the drag given by $\delta(v-\bar{v})$, where $\delta$ is the drag coefficient and $v$ and $\bar{v}$ are the velocities of the gas and solid phases, respectively. The rate of compaction is large immediately behind the leading shock due to the large difference in pressures of the two phases. A spike in the drag 

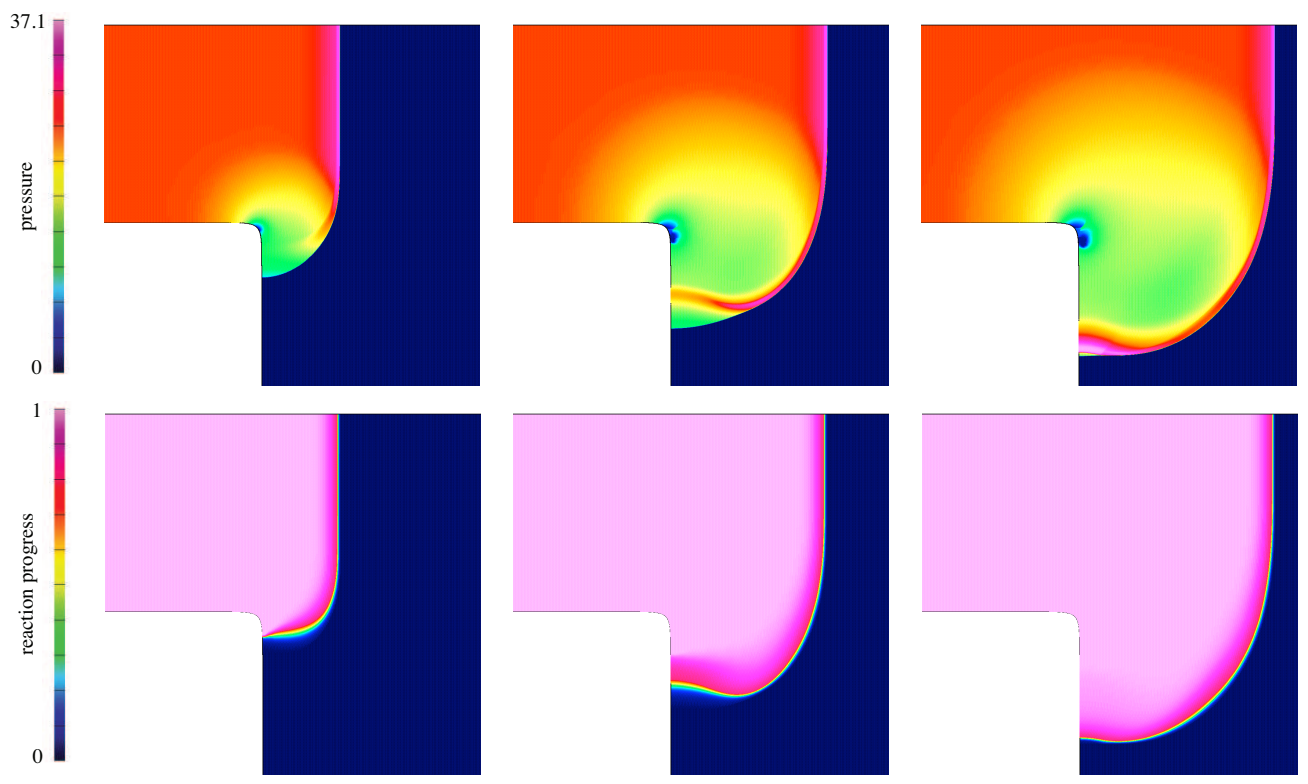

FIG. 4. Detonation diffraction for the IG model. Shaded contours of pressure in GPa (top row) and reaction progress (bottom row) for $t=0.4,0.8$ and $1.0 \mu$ s (left to right).

occurs at the leading edge of the reaction zone. This is a result of a velocity disequilibrium caused by the sudden increase in gas production due to chemical reaction behind the gas shock. The reaction rate, strongest at the gas shock, tails off as the solid phase is converted to gas. It is worth noting that the length of this steady "compaction-led" structure shown for the two-phase model is approximately three times greater than that shown for the IG model. This, and other features of the steady structures, depend on the choice of parameters for the two models. For example, other choices of the parameters for the two-phase model result in a "reaction-led" profile in which a shock in the gas phase and the reaction zone behind it lead the steady structure (see Schwendeman et al. (2007) for further details). Again, no attempt has been made to choose parameters to best match the solution behaviors of the two models. Instead, our focus is on the differences in the steady structures for representative sets of parameters, and how the structure of the detonation as determined by the two models plays a role in the subsequent diffraction and possible failure.

\subsection{Detonation diffraction}

We now turn our attention to the behavior of the detonation upon entering the acceptor charge in Figure 1 . For this discussion, we take $t=0$ to be the time when the steady detonation reaches the junction between the donor and acceptor charges. Figure 4 displays shaded contours of pressure $p$ and reaction progress $\lambda$ at three successive times for the IG model. The leading shock of the detonation appears in the plots of $p$ as a jump in color from deep blue for the ambient state to a warmer color depending on the strength of the shock. The reaction zone behind the leading shock is best viewed in the plots of $\lambda$, and it appears as a transition layer in color from deep blue (solid explosive) to pink (gaseous product). 

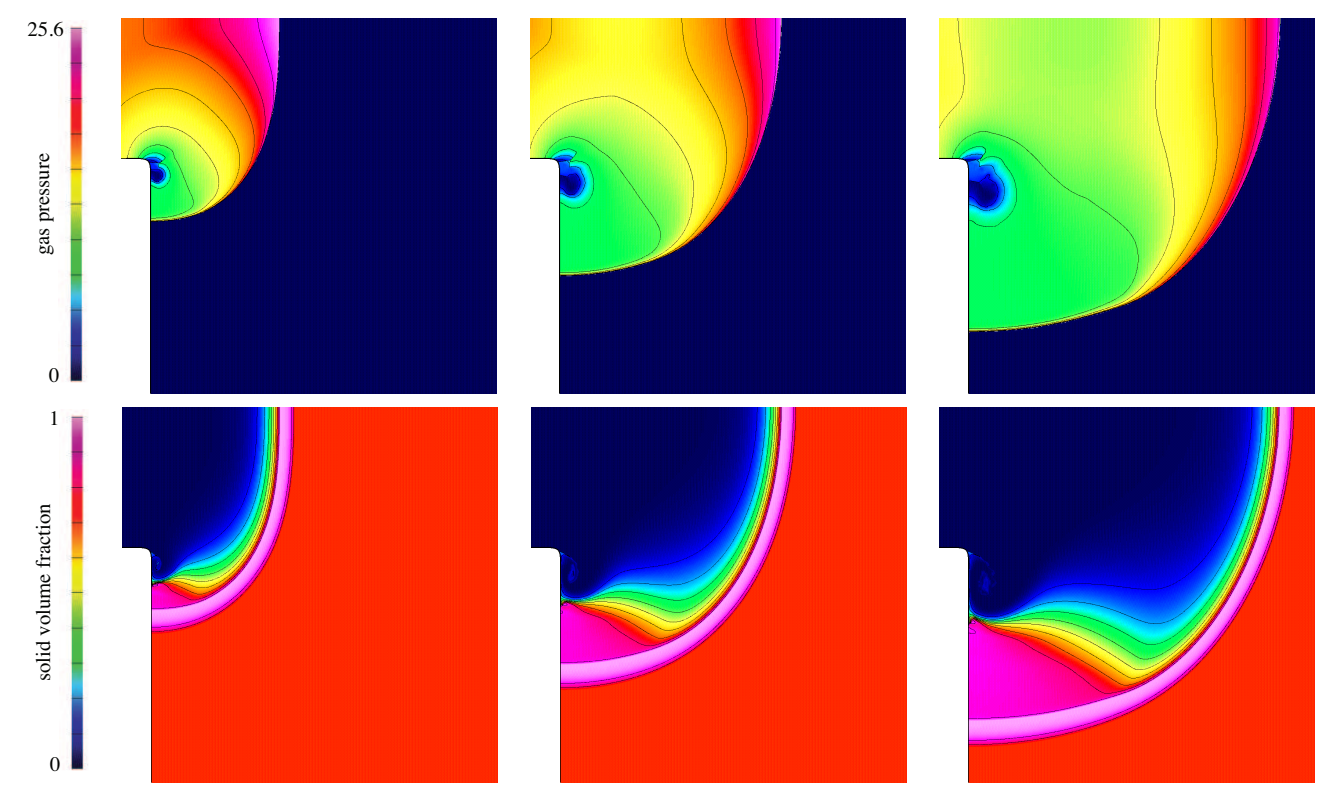

FIG. 5. Detonation diffraction for the two-phase model. Shaded contours of gas-phase pressure in GPa (top row) and solid volume fraction (bottom row) for $t=1.5,2.5$ and $3.5 \mu$ s (left to right).

At the earliest time, $t=0.4 \mu \mathrm{s}$, we observe that the leading shock has weakened along the vertical wall and the reaction zone is lagging behind it. As a result, there is a small region of compressed explosive in which the reaction there is weak or perhaps turned off altogether. At the next time shown, $t=0.8 \mu \mathrm{s}$, we note that a ridge of increased pressure has formed behind the leading shock. This ridge suggests that the reaction was not turned off, but rather was slowed and has now strengthened to create a pressure pulse similar to that observed in the evolution to detonation shown in the upper-left plot of Figure 2. The pressure pulse continues to strengthen with time and ultimately reaches the leading shock along the vertical wall by the final time shown, $t=1.0 \mu \mathrm{s}$. This process of self-ignition consumes the temporary pocket of unreacted explosive along the vertical wall, and ultimately leads to a diverging detonation in the acceptor charge with no sustained dead zone observed.

An analogous corner-turning simulation for the two-phase model is shown in Figure 5. In this figure, we show shaded contours of the gas-phase pressure $p$ (top row) and the solid volume fraction $\bar{\alpha}$ (bottom row) for three successive times. The plots of pressure show a diverging shock in the gas phase, and indicate regions behind the shock where $p$ is greater than $p_{\text {ign }}=10.2 \mathrm{GPa}$ so that the two-phase reaction rate is turned on. The plots of the solid volume fraction show a (pink) compaction layer at the front of the diverging detonation and a layer of decrease in $\bar{\alpha}$ through the reaction zone. At the earliest time shown, $t=1.5 \mu \mathrm{s}$, we observe that the compaction layer is thicker near the vertical wall and the gas shock behind it triggers only a very brief reaction. As a result, a small triangular-shaped pocket of partially-reacted explosive forms. As the detonation continues to diverge, this dead zone near the vertical wall grows, and there is no indication of any mechanism that would lead to a re-ignition of the partially-reacted material. The appearance of this sustained dead zone is qualitatively similar to that observed experimentally in Ferm et al. (2001). 

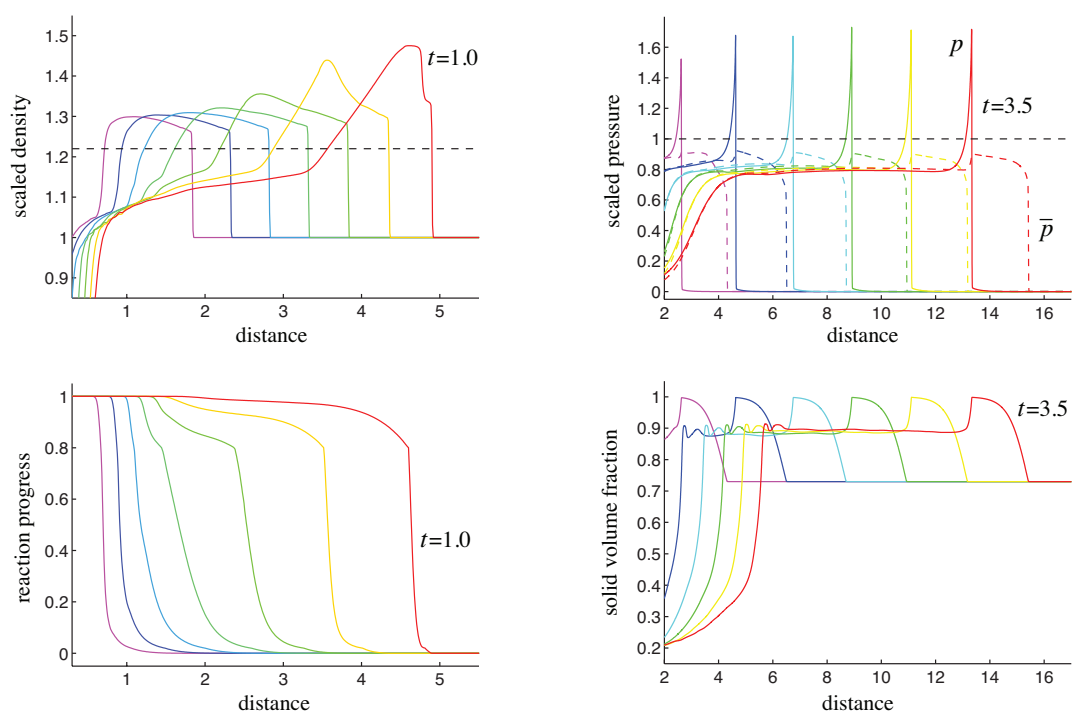

FIG. 6. Flow behavior along the vertical wall for the IG model (left) and the two-phase model (right). Distance along the wall is measured in $\mathrm{mm}$ from the $90^{\circ}$ corner. Density is scaled by $\rho_{0}=1905 \mathrm{~kg} / \mathrm{m}^{3}$ and pressure is scaled by $p_{\text {ign }}=10.2 \mathrm{GPa}$. The dashed black lines indicate reaction thresholds. The time interval between solution curves is $0.1 \mu \mathrm{s}$ for the IG case and $0.5 \mu \mathrm{s}$ for the two-phase case.

A closer look at the behavior of the flow along the vertical wall downstream of the corner provides a better understanding of the mechanisms leading to the re-birth of the detonation for the IG case and the sustained failure for the two-phase case. For the IG case, we show in Figure 6 the behavior of the scaled density $\rho / \rho_{0}$ and the reaction progress $\lambda$ for $t=0.4,0.5, \ldots, 1.0 \mu \mathrm{s}$. In the plot of density, we also indicate the value of the ignition threshold $1+a=1.22$ for the IG reaction rate in (2.4). At the earliest time shown, we see that while the leading shock is weak, it does provide a sufficient compression so that the scaled density jumps to a value above the ignition threshold behind the shock. Thus, the reaction is turned on behind the shock, but the rate is very slow as indicated by the near-zero values for $\lambda$ in the region immediately behind the shock. The gap between the leading shock and the fully reacted material behind it corresponds to the small pocket of unreacted material behind the shock observed in the shaded contour plots of Figure 4 for $t=0.4 \mu \mathrm{s}$. As time proceeds, we see that the scaled density begins to grow behind the shock indicative of a growing reaction rate there. This growth is seen clearly in the increasing values of $\lambda$ and leads to the re-birth of the detonation behind the leading shock. This behavior is similar to that discussed earlier for the impact problem and shown in Figure 2. The reborn detonation travels faster than the leading shock, and ultimately overtakes it leading to a detonation propagating down the vertical wall into the quiescent explosive. It is worth noting that for other corner-turning configurations, the scaled density behind the leading shock can fall below the ignition threshold in the post-diffracted region so that the reaction is turned off there, at least temporarily. In particular, for a $140^{\circ}$ corner it is found that re-ignition does not occur along the wall, but rather a detonation is born in a region away from the wall and then it propagates laterally to consume the unreacted material behind the leading shock near the wall. Again, the ultimate behavior is a diverging detonation in the acceptor charge with no sustained dead zone behind it (see Kapila et al. (2007) for further details). 
For the two-phase case, we show the behavior of the gas pressure $p$ (solid curves) and the solid pressure $\bar{p}$ (dashed curves) both scaled by $p_{\text {ign }}$ along the vertical wall in the upper-right plot of Figure 6 . Solution curves are plotted for $t=1.0,1.5, \ldots, 3.5 \mu \mathrm{s}$, and the ignition threshold $p / p_{\text {ign }}=1$ for the two-phase rate in (2.7) is also shown. The wave structure is led by a shock in the solid phase which is followed by a compaction in which $\bar{\alpha}$ increases from its ambient value to a value near one (as shown in the lower-right plot of the figure). This part of the wave structure is similar to that for the steady detonation structure shown in Figure 3, except that the jump in the solid pressure at the shock is much smaller (by a factor of nearly 4). There is a shock in the gas phase at the end of the compaction layer which has a peak value greater than the ignition threshold indicating that the reaction turns on. However, the gas pressure decreases rapidly from its peak value near 1.7 to a value below the ignition threshold. Thus, the reaction is on, but only for a very brief interval of time. The result is that the solid volume faction decreases abruptly from its near-one value at the rear of the compaction layer to a value of approximately 0.9 where the reaction turns off. This pocket of partially-reacted explosive along the wall grows with time, and there is no sign of any further mechanism of re-birth so that this material can be considered a sustained dead zone.

It is important to note that the behavior of the detonation in the post-diffracted region is very sensitive to the choice of the rate parameters for the two-phase model. For example, if the ignition pressure $p_{\text {ign }}$ is decreased by approximately $5 \%$ (with the other parameters held fixed), then it is found that the detonation is reborn in the region near the vertical wall and no sustained dead zones are observed (see Schwendeman et al. (2010)). The reason for this sensitivity is that the ignition threshold for the two-phase model is correlated to pressure, which tends to be a sensitive variable. The ignition threshold for the IG model, on the other hand, is correlated to density which is generally a less sensitive variable. Thus, the corner-turning behavior and absence of sustained dead zones is found to be robust feature of the IG model, while a wider range of behaviors are observed for the two-phase model.

\section{Concluding remarks}

The IG and two-phase models are well known macro-scale models of condensed-phase explosives. The IG model is representative of a homogeneous mixture model, while the more complicated two-phase model is one in which the solid and gas phases retain their constitutive identities. Detailed investigations of the behavior of solutions for a range of problems have been carried out for each model separately and these appear in the literature. The present discussion has focussed on a comparative study of the solutions for detonation initiation and diffraction. For detonation initiation it is found that while there are some similarities in the evolution and steady structures, the detailed mechanisms are different. The behavior determined for the IG model depends significantly on the assumed three-stage reaction law and no specific information regarding the interaction between the phases is given. The two-phase model, on the other hand, describes the interaction between the phases and this behavior plays a significant role in the evolution and structure of the detonation. Once a steady detonation is established, subsequent diffraction for the IG case shows weakening of the detonation and temporary failure, but no sustained dead zones. In contrast, solutions of the two-phase model may or may not show a sustained dead zone depending on the choice of parameters of the reaction rate law.

The results described here for the corner-turning geometry shown in Figure 1 assume rigid confinement. An investigation of detonation propagation and diffraction with compliant confinement is discussed in Banks et al. (2008) for the IG model, and it is found that the absence of rigid confinement does not affect the outcome of corner turning in a material way; sustained dead zones continued to elude the IG model. An analogous study of corner turning with compliant confinement for the two-phase 
model has not yet been carried out (to our knowledge), but such a study is under current investigation. Unlike the IG case, we believe that the behavior of the detonation in the post-diffracted region will be strongly affected by the choice of confinement due to the sensitive behavior of the gas pressure for the ignition-pressure rate law.

Dead zones observed in corner-turning experiments are believed to occur as a result of desensitization due to compaction of the explosive grains upon passage of the weakened shock in the post-diffracted region. The reaction rate in the standard IG model does not account for this desensitization, but an extension of the rate may be considered as is discussed, for example, in de Oliveira et al. (2006). In this paper, it is shown that sustained dead zones are possible for the IG case with the extended rate law that models shock desensitization explicitly. For the two-phase model, compaction of the solid is determined as part of the solution. The compaction of the solid, in turn, plays a role in the behavior of the gas pressure which is lied strongly to the rate of reaction. Thus, shock desensitization is incorporated implicitly into the two-phase model, and consequently sustained dead zones are observed even for the relatively simple ignition-pressure rate law assumed. In general, we believe that a higher fidelity in simulation of macro-scale behavior is possible for the two-phase model upon further calibration with experiment which may require additional tuning of the constitutive input and reaction rate law.

\section{Funding}

This work was supported by the National Science Foundation [grant DMS-1016188 to D.S. and A.K.]; Lawrence Livermore National Laboratory [subcontract B574692 to D.S.]; the U.S. Department of Energy [contract DE-AC52-07NA27344 to W.H.]; and with the support of the ASCR Applied Math Program of the DOE Office of Science.

\section{References}

BAer, M.R. \& Nunziato, J.W. (1986) A two-phase mixture theory for the deflagration-todetonation transition (DDT) in reactive granular materials, Int. J. Multiphase Flow, 12, 861-889.

Banks, J.B., Schwendeman, D.W., Kapila, A.K. \& Henshaw, W.D. (2007) A high-resolution Godunov method for multi-material flows on overlapping grids, J. Comput. Phys., 223, 262-297.

Banks, J.B., Schwendeman, D.W., Kapila, A.K. \& Henshaw, W.D. (2008) A study of detonation propagation and diffraction with compliant confinement, Combust. Theory Modeling, 12, 769808 .

Bdzil, J.B., Menikoff, R., Son, S.F., Kapila, A.K. \& Stewart, D.S. (1999) Two-phase modeling of deflagration-to-detonation transition in granular materials: A critical examination of modeling issues, Phys. Fluids, 11, 378-402.

Butler, P.B. \& KRIER, H. (1986) Analysis of deflagration-to-detonation transition in high-energy solid propellants, Combust. Flame, 63, 31-41.

de Oliveira, G., Kapila, A.K., Schwendeman, D.W., Bdzil, J.B., Henshaw, W.D. \& TARVER, C.M (2006) Detonation diffraction, dead zones, and the ignition-and-growth model. In: The Thirteenth Symposium (International) on Detonation. 
Ferm, E., Morris, C., Quintana, J., Pazuchanic, P., Stacy, H., Zumbro, J., Hogan, G. \& KING, N. (2001) Proton radiography examination of unburned regions in PBX-9502 corner turning experiments, Tech. report LA-UR-01-3555, Los Alamos National Laboratory.

Henshaw, W.D. \& Schwendeman, D.W. (2003) An adaptive numerical scheme for high-speed reactive flow on overlapping grids, J. Comput. Phys., 191, 420-447.

Henshaw, W.D. \& Schwendeman, D.W. (2008) Parallel computation of three-dimensional flows using overlapping grids with adaptive mesh refinement, J. Comput. Phys., 227, 7469-7502.

Kapila, A.K., Son, S.F., Bdzil, J.B., Menikoff, R. \& Stewart, D.S. (1997) Two-phase modeling of DDT: Structure of the velocity-relaxation zone, Phys. Fluids, 9, 3885-3897.

Kapila, A.K., Menikoff, R., BdzIL, J.B., Son, S.F. \& Stewart, D.S. (2001) Two-phase modeling of deflagration-to-detonation transition in granular materials: Reduced equations, Phys. Fluids, 10, 3002-3024.

Kapila, A.K., Schwendeman, D.W., Bdzil, J.B. \& Henshaw, W.D. (2007) A study of detonation diffraction in the ignition-and-growth model, Combust. Theory and Modeling, 11, 781-822.

LEE, E.L \& TARVER, C.M. (1980) Phenomenological model of shock initiation in heterogeneous explosives, Phys. Fluids, 23, 2362-2372.

Powers, J.M., Stewart, D.S. \& Krier, H. (1990) Theory of two-phase detonation, Part I: Modeling, Combust. Flame, 80, 264.

Powers, J.M., StewArt, D.S. \& KRIER, H. (1990) Theory of two-phase detonation, Part II: Structure, Combust. Flame, 80, 280.

SAurel, R \& ABgrall, R. (1999) A multiphase Godunov method for compressible multifluid and multiphase flows, J. Comput. Phys., 150, 425-467.

SAurel, R \& Lemetayer, O. (2001) A multiphase model for compressible flows with interfaces, shocks, detonation waves and cavitation, J. Fluid Mech., 431, 239-271.

Schwendeman, D.W., Wahle, C.W. \& Kapila, A.K. (2006) The Riemann problem and a highresolution Godunov method for a model of compressible two-phase flow, J. Comput. Phys., 212, 490526.

Schwendeman, D.W., Wahle, C.W. \& Kapila, A.K. (2007) A study of detonation evolution and structure for a model of compressible two-phase reactive flow, Combust. Theory and Modeling, 12, 159-204.

Schwendeman, D.W., Kapila, A.K. \& Henshaw, W.D. (2010) A study of detonation diffraction and failure for a model of compressible two-phase reactive flow, Combust. Theory and Modeling, 14, 331-366.

Souers, P.C., Garza, R.\& Vitello, P. (2002) Ignition and growth and JWL++ detonation models in course zones, Propellants, Explosives and Pyrotechnics, 27, 62-71.

TARVER, C.M. (2005) Ignition-and-growth modeling of LX-17 hockey puck experiments, Propellants, Explosives and Pyrotechnics, 30, 109-117. 
FicketT, W. \& DAVIs, W.C. (1979) Detonation. Berkeley: University of California Press. 